\title{
A Genetically Optimized Level Set Approach to Segmentation of Thyroid Ultrasound Images
}

\author{
Dimitris K. Iakovidis, Michalis A. Savelonas, Stavros A. Karkanis, \\ Dimitris E. Maroulis
}

\begin{abstract}
This paper presents a novel framework for thyroid ultrasound image segmentation that aims to accurately delineate thyroid nodules. This framework, named GA-VBAC incorporates a level set approach named Variable Background Active Contour model (VBAC) that utilizes variable background regions, to reduce the effects of the intensity inhomogeneity in the thyroid ultrasound images. Moreover, a parameter tuning mechanism based on Genetic Algorithms (GA) has been considered to search for the optimal VBAC parameters automatically, without requiring technical skills. Experiments were conducted over a range of ultrasound images displaying thyroid nodules. The results show that the proposed GA-VBAC framework provides an efficient, effective and highly objective system for the delineation of thyroid nodules.

Keywords: Level Sets, Active Contour Models, Genetic Algorithms, Segmentation, Thyroid, Ultrasound.
\end{abstract}

\section{Introduction}

The inherent inhomogeneity of ultrasound medical images poses a challenge to image analysis researchers in developing accurate segmentation methods for ascertaining the shape features of tissue masses within living organisms. Despite the advantages of ultrasonic imaging in medicine [1], the images produced are highly distorted by noise and speckle [2], whereas their clinical assessment is subject to the radiologists' expertise. A frequent task assigned to radiologists is the assessment of nodules in thyroid ultrasound images. Thyroid nodules are solid or cystic lumps formed in the thyroid gland. Their prevalence increases with age and they afflict more than $50 \%$ of 
the world's population [3]. The echogenicity, the size and the shape of the thyroid nodules comprise a set of malignancy risk factors that should always be considered by the clinicians before proceeding to fine needle aspiration biopsy. Epidemiological studies have showed that hypo-echoic nodules with irregular borders are more likely to be or to evolve into malignant tumors [4]. Therefore, a computational model that could accurately delineate thyroid nodules would definitely be an aid, even to experienced radiologists, by providing a second opinion for the characterization of nodules based on explicit image features.

In this paper we propose a novel framework that aims to accurately delineate thyroid nodules in ultrasound images. It incorporates an image segmentation approach that reduces the effects of the intensity inhomogeneity in the thyroid ultrasound images and a Genetic Algorithm (GA) that automatically tunes the parameters involved.

The rest of this paper is organized in six sections. Section 2 reviews previous ultrasound image segmentation approaches. Section 3 describes the segmentation approach we have developed. The proposed framework for automatic delineation of thyroid nodules is described in Section 4. The results from the application of the proposed framework on real thyroid ultrasound images are apposed in Section 5. Finally, Section 6 summarizes the conclusions of this study and suggests future research perspectives.

\section{Ultrasound Image Segmentation}

Image segmentation approaches that have been proposed for the delineation of objects in ultrasound images, include thresholding [5], region growing [6]-[8], classification [9], clustering [10], wavelet analysis [11], mathematical morphology [12], genetic and fuzzy algorithms [13],[14].

Simple intensity thresholding may be sufficient for the segmentation of images containing regions with intensity values that exhibit small variance and do not overlap with the intensity values of another region. A more sophisticated thresholding

approach developed for the segmentation of ultrasound images that contain 
homogeneous regions, has been proposed in [5]. It employs a linear combination of gray level and local entropy for the delineation of ovarian cysts surrounded by soft tissue. The cysts contain transparent fluid and appear as dark homogeneous regions characterized by a narrow gray level distribution. The soft tissue appears bright and has a much wider intensity distribution. However, ultrasound images commonly contain inhomogeneous regions which may cause thresholding approaches to fail [15].

Region growing image segmentation methods usually involve the definition of an initial set of seed pixels and the growing of a uniform and connected region from each seed, according to an appropriately selected homogeneity criterion [6]-[8]. Their main advantage is that they are insensitive to local perturbations as they test the statistics inside the region. Approaches of this class are sufficient for the segmentation of homogeneous image regions. Ultrasound images contain speckle noise, tissue texture and artifacts which may lead to boundary discontinuities, small holes in the delineated regions, as well as to the delineation of incorrect regions [16],[17]. Moreover, the resulting segmentation depends on the choice of seed pixels [18], and human interaction may be required [19].

Image feature extraction approaches followed by classification or clustering of the resulting feature vectors have also been applied for the segmentation of ultrasound images. The methodology described in [9], involves the extraction of simple graylevel histograms from square regions of ultrasound images, and their classification by an SVM classifier. The size of the considered square regions is selected empirically and the optimal values of the SVM parameters are determined according to [20]. The resulting feature vectors are large, leading to a rather time consuming segmentation phase.

A k-means clustering approach that utilizes features derived from the wavelet transform coefficients of the image for the segmentation of breast ultrasound images has been proposed in [10]. This clustering approach can be time efficient [21] but it involves empirically determined parameters and, just like thresholding and region growing approaches, it tends to fail to segment images that contain inhomogeneous regions [6]. 
Noise reduction via pre-processing, is commonly necessary before the application of the previously described methods. In [11] it is shown that the segmentation performance could be enhanced by nonlinear wavelet filtering of the ultrasound images followed by soft thresholding of the wavelet coefficients.

Morphological filtering involves the modification of the spatial form or structure of the objects within an image. Its applications include the segmentation of ultrasound images for the measurement of the fetal femur length and the estimation of the gestational age [12]. This method preserves the shape information of the objects of interest but it requires additional edge-linking operations to improve the connectivity of the resulting contours.

In [13], a genetic algorithm applies for the search of contours in ultrasound images of human legs. This algorithm assumes that the contours are modeled by closed cubic splines and searches for contours that best fit to this model. It accomplishes automatic image segmentation and avoids noise diversions; however, it converges to contour solutions that are constrained by the particular model.

A fuzzy approach to segmentation of ultrasound images has been proposed in [14]. This method applies fuzzy logic for edge detection using appropriately selected edge membership functions. As with morphological filtering, edge-linking post processing is necessary for obtaining continuous contours.

The application of active contour models for ultrasound image segmentation has been gaining increasing interest over the previously described methods. They are selfadapting models that lead to continuous, closed or open, curves without requiring edge-linking operations. Active contour models span two categories: parametric and level set. They both share a common underlying idea: initial contours deform towards the boundaries of image regions with common features. Parametric active contour models are local methods based on an energy-minimizing spline guided by external and image forces, which pull or push the spline toward features such as lines and edges in the image. Level set active contours are also energy minimization techniques that solve the computation of geodesics or minimal distance curves [22]. They can be used for the delineation of multiple objects in an image, whereas the parametric active contours are not so flexible to allow topological changes of the contour during its evolution. The utility of the level set approaches in medical image segmentation is 
becoming clear in various medical applications such as in the automatic quantification of the ventricular function [23], in prostate [24] and in cardiac ultrasound image segmentation [25].

A state of the art level set approach to image segmentation is the Active Contour Without Edges (ACWE) model [26]. ACWE can be relatively insensitive to noise, as it involves integral operators, which provide an inherent noise filtering mechanism [15]. Therefore, it does not require any noise reduction pre-processing of the input images, the boundaries of the objects of interest are preserved and can be accurately detected. Moreover, the ACWE model does not impose any significant initialization constraint and allows the detection of objects whose boundaries are either smooth or not necessarily defined by gradient. In such cases the standard active contour models commonly fail and result in boundary leakage. Although the ACWE model can be advantageous for ultrasound image segmentation compared with the previously described methods, it is sensitive to intensity inhomogeneity leading to less accurate segmentation.

Motivated by the ACWE model [26] we developed an improved level set approach named Variable Background Active Contour model (VBAC). This model utilizes variable background regions, to reduce the effects of the intensity inhomogeneity in the thyroid ultrasound images, which include speckle noise, tissue texture and calcifications. The improved performance of VBAC over the ACWE model for the delineation of thyroid nodules has been demonstrated in [27].

A drawback in the application framework of active contour models to ultrasound images, including that of VBAC, is that it is device dependent; meaning that for the segmentation of images acquired from different ultrasound imaging devices, or from the same imaging device using different settings (e.g. dynamic range), a set of different parameter values is required. In most cases parameter tuning requires technical skills and time-consuming manual interaction, which could hardly be performed by physicians. Hence, the routine use of active contour models in medical image segmentation has been so far limited. The requirement of manual interaction can be overcome if we consider automatic parameter tuning.

Automatic parameter tuning has received very little attention in active contour literature, even though the choice of the parameters can be critical for the image 
segmentation performance. For example, in [28],[29] the tuning of the model parameters relies basically on empirical observations. There has been only a limited number of studies that deal with the problem of parameter tuning in active contour models, and these include a bound setting approach [30], an unsupervised method based on a minimax criterion [31], and GAs as a supplement to Taguchi approach for fine-tuning the parameters of a parametric active contour model for cardiac ultrasound image segmentation [32].

In order to cope with the automatic tuning of VBAC parameters so as to obtain accurate delineation of thyroid nodules, we introduce a novel framework that combines VBAC with a GA. The proposed framework will be referred to as GAVBAC.

\section{Level Set Formulation}

\subsection{Active Contour Without Edges}

The ACWE model as posed in [26] has the form of a minimization problem: Let $\Omega$ be a bounded open subset of $R^{2}$ and $\partial \Omega$ its boundary. We seek for the infimum of the energy functional $F\left(c^{+}, c^{-}, C\right)$,

$$
\begin{aligned}
F\left(c^{+}, c^{-}, C\right) & =\mu \cdot \operatorname{Length}(C) \\
& +\lambda^{+} \int\left|u_{0}(x, y)-c^{+}\right|^{2} d x d y \\
& +\left.\lambda_{\text {inside }(C)}^{-} \int_{\text {ousside( }(C)}^{\int \mid u_{0}(x, y)-c^{-}}\right|^{2} d x d y
\end{aligned}
$$

where $u_{0}: \Omega \rightarrow R$ is the input image, $C(s):[0,1] \rightarrow R^{2}$ is a piecewise parameterized curve, $c^{+}$and $c^{-}$represent the average value of $u_{0}$ inside and outside the curve and parameters $\mu>0$ and $\lambda^{+}, \lambda^{-}>0$ are weights for the regularizing term and the fitting terms, respectively. This problem is a special case of the minimal partition problem, for which the existence of minimizers has been proved in [33]. As in the minimum 
energy problem, the minimizer corresponds to the "equilibrium" of the regularizing and fitting terms that force the contour to stop.

In the level set method [34], $C \subset \Omega$ is represented by the zero level set of a Lipschitz function $\phi: \Omega \rightarrow R$, such that

$$
\begin{aligned}
& C=\{(x, y) \in \Omega: \phi(x, y)=0\}, \\
& \text { inside }(C)=\{(x, y) \in \Omega: \phi(x, y)>0\}, \\
& \text { outside }(C)=\{(x, y) \in \Omega: \phi(x, y)<0\}
\end{aligned}
$$

Using the one-dimensional Dirac function $\delta$ and the Heaviside function $H$, which are defined respectively by

$$
\delta(z)=\frac{d}{d z} H(z), H(z)= \begin{cases}1, & \text { if } z \geq 0 \\ 0, & \text { if } z<0\end{cases}
$$

where $z \in R$, the average foreground and background intensities $c^{+}$and $c^{-}$can be determined by

$$
\begin{gathered}
c^{+}(\phi)=\frac{\int_{\Omega} u_{0}(x, y) H(\phi(x, y)) d x d y}{\int_{\Omega} H(\phi(x, y)) d x d y} \\
c^{-}(\phi)=\frac{\int_{\Omega} u_{0}(x, y)(1-H(\phi(x, y))) d x d y}{\int_{\Omega}(1-H(\phi(x, y))) d x d y} .
\end{gathered}
$$

By keeping $c^{+}$and $c^{-}$fixed, and minimizing $F$ with respect to $\phi$, the associated Euler-Langrange equation for $\phi$ is deduced. For this purpose, slightly regularized versions of $H$ and $d$ are considered. The regularized Heaviside function $H_{\varepsilon}$ is a continuously differentiable function for all degrees of differentiation and it is derived as follows

$$
H_{\varepsilon}(z)=\frac{1}{2} \cdot\left(1+\frac{2}{\pi} \arctan \left(\frac{z}{\varepsilon}\right)\right)
$$


whereas the corresponding regularized delta function $\delta_{\varepsilon}$ is derived from $\delta_{\varepsilon}=d H_{\varepsilon} / d z$. As $\varepsilon \rightarrow 0$, both approximations converge to $H$ and $d$. These approximations allow the algorithm to compute a global minimizer, as described in [26].

Parameterizing the descent direction by an artificial time $t \geq 0, \phi(t, x, y)$, (with $\phi(0, x, y)=\phi_{0}(x, y)$ defining the initial contour $)$ is determined by the following equation

$$
\frac{\partial \phi}{\partial t}=\delta(\phi)\left[\mu \cdot \operatorname{div}\left(\frac{\nabla \phi}{|\nabla \phi|}\right)-\lambda^{+}\left(u_{0}-c^{+}\right)^{2}+\lambda^{-}\left(u_{0}-c^{-}\right)^{2}\right]=0
$$

where $\operatorname{div}(\overrightarrow{\mathbf{V}})=\vartheta V_{1} / \vartheta x+\vartheta V_{2} / \vartheta y$ is the divergence of a vector $\overrightarrow{\mathbf{V}}=\left(V_{1}, V_{2}\right)$ and $t \in(0, \infty),(x, y) \in \Omega$,

A discretized and linearized version of (7) is:

$$
\begin{aligned}
& \frac{\phi_{i, j}^{n+1}-\phi_{i, j}^{n}}{\Delta t}=\delta\left(\phi_{i, j}^{n}\right) \cdot\left[\mu K-\lambda^{+}\left(u_{0, i, j}-c^{+}\left(\phi^{n}\right)\right)^{2}+\lambda^{-}\left(u_{0, i, j}-c^{-}\left(\phi^{n}\right)\right)^{2}\right] \Rightarrow \\
& \phi_{i, j}^{n+1}=\Delta t \cdot\left[\phi_{i, j}^{n}+\delta\left(\phi_{i, j}^{n}\right) \cdot\left[\mu K-\lambda^{+}\left(u_{0, i, j}-c^{+}\left(\phi^{n}\right)\right)^{2}+\lambda^{-}\left(u_{0, i, j}-c^{-}\left(\phi^{n}\right)\right)^{2}\right]\right]
\end{aligned}
$$

where $\Delta t$ is the time step, $\left(x_{i}, y_{j}\right)$ are the grid points for $1 \leq i, j \leq M, M$ represents the number of points on each side of grid, $\phi_{i, j}^{n}=\phi\left(n \Delta t, x_{i}, y_{j}\right)$ and $u_{0, i, j}=u_{0}\left(x_{i}, y_{j}\right)$ are approximations of $\phi(t, x, y)$ and $u_{0}(x, y)$ respectively, with $n \geq 0$. The curvature $K$ of $\phi$ is obtained from

$$
K=\operatorname{div}\left(\frac{\nabla \phi}{|\nabla \phi|}\right)=\frac{\frac{\partial^{2} \phi}{\partial x^{2}} \cdot\left(\frac{\partial \phi}{\partial y}\right)^{2}-2 \frac{\partial \phi}{\partial x} \cdot \frac{\partial \phi}{\partial y} \cdot \frac{\partial^{2} \phi}{\partial x \partial y}+\frac{\partial^{2} \phi}{\partial y^{2}} \cdot\left(\frac{\partial \phi}{\partial x}\right)^{2}}{\left(\left(\frac{\partial \phi}{\partial x}\right)^{2}+\left(\frac{\partial \phi}{\partial y}\right)^{2}\right)^{\frac{3}{2}}}
$$

where $\partial \phi / \partial x, \partial \phi / \partial y$ are calculated by $\Delta^{x} \phi_{i, j} / \Delta s, \Delta^{y} \phi_{i, j} / \Delta s, \Delta^{x} \phi_{i, j}=\phi_{i+1, j}-\phi_{i, j}$, $\Delta^{y} \phi_{i, j}=\phi_{i, j+1}-\phi_{i, j}$ and $\Delta s$ is the step between two consecutive grid points of the discrete space.

In a practical implementation, a criterion should force the algorithm to stop when a stationary solution is reached. This criterion can be expressed as follows: 


$$
Q \leq(\Delta t) \cdot(\Delta s)^{2}
$$

where

$$
Q=\frac{\sum_{\phi_{i, j}^{n}, k \Delta s}\left|\phi_{i, j}^{n+1}-\phi_{i, j}^{n}\right|}{M^{\prime}}
$$

and $M^{\prime}$ is the number of grid points for which $\left|\phi_{i, j}^{m}\right|<\Delta s$.

A limitation of the ACWE model is that it assumes approximately piecewise constant intensities for object and background regions. This assumption is violated in thyroid US images where intensity inhomogeneities are present in the background image region with a consequent negative impact on the delineation of hypo-echoic nodules.

\subsection{Variable Background Active Contour Model}

Aiming to surpass the limitation of the ACWE model resulting from its assumption of approximately piecewise constant image intensities and in order to enhance the delineation accuracy of hypo-echoic nodules in the presence of intensity inhomogeneities, we introduce the Variable Background Active Contour (VBAC) model. This model utilizes a variable background from which inhomogeneities are excluded. The remaining background regions are expected to be approximately constant and thus the assumption of constant background intensities used in the ACWE model is better approximated.

The intensity inhomogeneity affects $c^{-}$, which is defined in (5) as the average background intensity. The VBAC model incorporates a new $c^{-}$which is calculated as the average of the remaining background from which inhomogeneity regions are excluded.

We define the difference $D(\phi(x, y))$ as

$$
D(\phi(x, y))=H(\phi(x, y)+a)-H(\phi(x, y))
$$

where $\alpha$ is a positive constant. Its value is determined so that $[-a, 0]$ defines the acceptable range of $\phi(x, y)$ for a point $(x, y)$ to be included in the variable 
background. Equations (6) and (12) imply that the points $(x, y)$ for which $\phi(x, y) \notin[-a, 0]$ result in $D(\phi(x, y)) \approx 0$. These points that correspond to intensity inhomogeneities within the region of interest, cause abrupt changes of $\phi$, and result in $H(\phi(x, y)+a)=H(\phi(x, y))$. Moreover, we assume that the initial contour as traced by $\phi_{0}$ corresponds to a region of interest within the thyroid gland and we employ $H\left(\phi_{0}\right)$ to restrict the calculation of the average foreground and background intensities $c^{+}$ and $c^{-}$over this region. Equations (4) and (5) are reformulated as follows

$$
\begin{gathered}
c^{+}(\phi)=\frac{\int_{\Omega} u_{0}(x, y) H(\phi(x, y)) H\left(\phi_{0}(x, y)\right) d x d y}{\int_{\Omega} H(\phi(x, y)) H\left(\phi_{0}(x, y)\right) d x d y} \\
c^{-}(\phi)=\frac{\int_{\Omega} u_{0}(x, y)(1-H(\phi(x, y))) H\left(\phi_{0}(x, y)\right) D(\phi(x, y)) d x d y}{\int_{\Omega}(1-H(\phi(x, y))) H\left(\phi_{0}(x, y)\right) D(\phi(x, y)) d x d y} .
\end{gathered}
$$

Equation (14) imposes that a point $(x, y) \in \Omega$ is not included in the calculation of $c^{-}$ if $D(\phi(x, y))=0$. The VBAC model is finally described by (7),(12)-(14).

\section{[FIGURE 1]}

The VBAC algorithm can be summarized as follows

Step 1. Initialize $n \leftarrow 0, \phi^{0} \leftarrow \phi_{0}$

Step 2. Calculate $D\left(\phi^{n}\right)$ by (12)

Step 3. Calculate $c^{+}\left(\phi^{n}\right)$ and $c^{-}\left(\phi^{n}\right)$ by (13) and (14)

Step 4. Calculate $\phi^{n+1}$ by (8)

Step 5. If inequality (10) is true Then End

Step 6. $n \leftarrow n+1$

Step 7. Repeat Steps 2 to 6. 
As the algorithm proceeds the term $c^{-}$varies, resulting in a variable background. For example, Fig. 1(a) illustrates a snapshot of the contour, Fig. 1(b) the pixels considered as background and Fig. 1(c) the function $\phi(x, y)$ at a random iteration of the algorithm. The sparsity of the background in Fig. 1(b) reveals the selectivity of VBAC. Pixels belonging to calcifications and other inhomogeneities in the thyroid ultrasound image have been excluded from the background. The 3-dimensional diagram illustrated in Fig. 1(c) displays $\phi(x, y)$ crossing the zero-level plane on which the image resides. The active contour is formed by the set of pixels belonging to the cross-section of $\phi(x, y)$ with this plane.

\section{Genetic Optimization Framework}

A naive approach to tuning the four VBAC parameters, namely $\lambda^{+}, \lambda^{-}, \mu$ and $\alpha$, for segmentation of images acquired from an ultrasonic imaging device, is the exhaustive search of all possible solutions in a discretized parameter space. Such an approach can lead to optimal parameter values but it is time consuming, to an extent that it could be prohibiting for medical application on routine basis. Also, many researchers in the field of active contour applications commonly employ empirical

approaches to parameter tuning [26],[28],[29]. However, such approaches lack scientific foundation, lead to suboptimal solutions, and require specialized technical knowledge and experience, that could hardly be found in a physician's background.

The GA-VBAC framework proposed in this paper aims to efficient parameter tuning of VBAC, based on Genetic Algorithms (GAs). GAs are stochastic non-linear optimization algorithms based on the theory of natural selection and evolution [35],[36]. They are similar to traditional search techniques such as simulated annealing [37], but they differ in that they are parallelized, maintaining a population of solutions from which new solutions are generated. Moreover, GAs perform crossover operations which generate new solutions by combining existing solutions from the solution pool, allowing the algorithm to "jump" within the optimization 
landscape. GAs have been the optimizers of choice in various artificial intelligence applications, exhibiting better performance than other non-linear optimization approaches to parameter tuning. Such applications include parameter tuning in support vector machines [38],[39], tuning of neural network weights for on-line training [40], tuning of range image segmentation algorithms [41], and the fine-tuning of a parametric active contour model which is supplementary to the Taguchi approach [32].

Motivated by these studies, we transcribed the parameter tuning optimization problem of the level-set VBAC model into a genetic optimization problem. Considering that $\mu, \lambda^{+}, \lambda^{-}$are weight terms of the energy functional that regulate the relative influence of the terms comprising (1), and that $\mu>0$, (7) can be rewritten as follows

$$
\delta(\phi)\left[\operatorname{div}\left(\frac{\nabla \phi}{|\nabla \phi|}\right)-\frac{\lambda^{+}}{\mu}\left(u_{0}-c^{+}\right)^{2}+\frac{\lambda^{-}}{\mu}\left(u_{0}-c^{-}\right)^{2}\right]=0
$$

and by setting $k^{+}=\frac{\lambda^{+}}{\mu}$ and $k^{-}=\frac{\lambda^{-}}{\mu},(15)$ can be rewritten as follows

$$
\delta(\phi)\left[\operatorname{div}\left(\frac{\nabla \phi}{\nabla \phi \mid}\right)-k^{+}\left(u_{0}-c^{+}\right)^{2}+k^{-}\left(u_{0}-c^{-}\right)^{2}\right]=0 .
$$

The parameters $k^{+}, k^{-}$and $\alpha$ are encoded into a single bit-string, called chromosome. Their values are constrained within discrete, worst-case ranges. The minimum and maximum values of these ranges as well as the sampling rate considered have been experimentally determined. So, $k^{+}$and $k^{-}$are represented by 8 bit variables with values ranging from 0 to 255 , whereas a 4-bit variable is used to hold the exponents of $\alpha$, which enumerates the values $10^{-15}, 10^{-14}, \ldots, 10^{0}$. The length of the resulting chromosome sums a total of 20-bits (Fig. 2).

\section{[FIGURE 2]}

The GA-VBAC framework is a supervised approach searching for the optimal parameters $\left(k^{+}, k^{-}, \alpha\right)$ that maximize the overlap value $f$ between a contour $A$ and a 
given ground truth delineation $T$ of a thyroid nodule. The ground truth delineation comprises of all pixels falling within at least $N / 2+1$ out of $N$ delineations drawn by $N$ expert radiologists [42]. The bias introduced in the ground truth delineation is reduced as $N$ increases. The overlap value $f$ between two delineated areas $A$ and $T$ is defined as in [8]

$$
f=\frac{A \cap T}{A \cup T} .
$$

In case of a perfect match between the two delineated areas $A$ and $T$, the overlap value is maximized $(f=1)$.

The GA of the proposed framework proceeds to the reproduction of an initial population of $R$ chromosomes by following the steady state approach. According to this approach the fittest individuals, i.e. the chromosomes that lead to large overlap values $f$, are maintained in the population of the generations $G$ produced by the successive iterations of the algorithm. Comparative studies have showed that steady state approaches can find solutions which are as good as, or better than solutions generated by generational GAs in much less time [43]. The selection of the individuals to survive is based on the tournament approach: a random subpopulation of $q>1$ individuals is taken from the population and the best of these $q$ individuals is selected to survive for the next generation [44]. This method was chosen as a computationally efficient alternative, and allows for fine-tuning the selective pressure by increasing or decreasing the tournament size $q$. The selection is repeated until all the individuals of the current population take part in exactly one tournament.

The winner individuals of all tournaments are maintained in the population and they are used to generate offspring individuals by multi-parent diagonal crossover. The multi-parent diagonal crossover operator exchanges segments from the selected individuals (parents) to generate offspring individuals. Diagonal crossover has been introduced as a generalization of 1-point crossover. It generates $r$ offsprings from $r$ parents by selecting $(r-1)$ crossover points in the parents and composing the offsprings by taking the resulting $r$ chromosome segments from each parent along the diagonals (Fig. 3). This approach commonly yields a performance improvement over 
single-parent crossover operators whereas it lowers the probability of premature convergence [45],[46].

[FIGURE 3]

Following crossover, a mutation operator is applied, flipping the bit content of the chromosomes at random positions from 1 to 0 , and vice versa, usually with very low probability [47]. Mutation provides a mechanism to keep the solution away from local minima [32]. The offspring individuals generated by crossover and mutation replace the individuals of the population that lost in the preceding tournament. The GA will eventually converge because the fittest individual will take part in exactly one tournament and it will be maintained in the population until the algorithm ends after $G_{\max }$ generations [48].

\section{[FIGURE 4]}

Figure 4 illustrates a flowchart of the GA-VBAC framework. The algorithm proceeds as follows

Step 1. Initialize $G \leftarrow 0, f_{\text {FITTEST }} \leftarrow 0$

Generate Population of $R$ Chromosomes at random

Step 2. For each Chromosome

Execute VBAC on input image

Calculate $f(G)$

If $f(G) \geq f_{\text {FITTEST }}$ Then

$$
f_{\text {FITTEST }} \leftarrow f(G)
$$

Register $f_{\text {FITTEST }}$

End If

End For

Step 3. $G \leftarrow G+1$

Step 4. Begin Reproduction

Select fittest Chromosomes

Maintain fittest Chromosomes in the Population 


\section{End Reproduction}

Step 5. Crossover fittest Chromosomes to Generate new Chromosomes

Step 6. Mutate Fittest Chromosomes to Generate new Chromosomes

Step 7. Repeat Steps 2 to 6 Until $G=G_{\max }$

The GA-VBAC training procedure, described above, will result in a registered optimal set of parameters $\left(k^{+}, k^{-}, \alpha\right)$. This set of parameters can be used for the delineation of nodules in other thyroid ultrasound images acquired from the same ultrasound imaging device with the same settings.

\section{Results}

Extensive experimentation was performed over real ultrasound images, in order to evaluate the performance of the proposed GA-VBAC framework for the delineation of thyroid nodules, and to compare its results with those obtained by individual expert radiologists.

A total of 71 longitudinal in vivo digital images of various thyroid nodule cases were acquired at a resolution of $256 \times 256$ pixels with a 256 grey-level depth, using a digital ultrasound imaging system HDI 3000 ATL with a 5-12 MHz linear transducer. The dataset used in the experiments comprised of 45 images of hypo-echoic nodule cases. The images were all acquired with fixed settings for the ultrasound imaging system. Three expert radiologists $(N=3)$ manually delineated each thyroid nodule in the ultrasound images of the dataset and ground truth delineations were obtained by following the rule described in Section 4.

The GA-VBAC framework was implemented in Microsoft Visual $\mathrm{C}++$ and executed on a $3.2 \mathrm{GHz}$ Intel Pentium IV workstation. The GA-VBAC parameters were kept constant during the experimentation. A typical population of $R=30$ chromosomes was considered in agreement with [49]. A typical value of $q=2$ was used in the tournament selection process. The crossover probability $p_{c}$ was set at 0.6 [50] and the mutation probability $p_{m}$ was set at $1 / l=0.05$, where $l=20$ is the 
chromosomes' length [46]. A number of 50 generations was considered as $G_{\max }$ as it allows for convergence to the highest attainable fitness value.

The GA-VBAC framework accepts a single image for training (Fig. 4). In order to avoid the sample selection bias that would be introduced if the performance evaluation process used a single training image, arbitrarily selected from the available set of images, a cross-validation scheme was employed. This scheme involved a total of 45 experiments that use independent training and testing images. In each experiment, a different image was drawn from the dataset and used for training whereas the rest 44 images of the dataset were used for testing.

The ranges of the overlap values (f-ranges) obtained by the automated GA-VBAC framework and by the experts with respect to the ground truth delineations per case are illustrated in Fig. 5. The bold vertical bars indicate the $f$-ranges corresponding to the GA-VBAC framework, as obtained by the test results of the cross validation process. The plain vertical bars indicate the ranges between the minimum and the maximum overlap values obtained by the expert radiologists.

\section{[FIGURE 5]}

Figure 5, shows that in 22 cases, GA-VBAC leads to $f$-ranges that fall within the experts' $f$-ranges. In 17 cases it leads to $f$-ranges that exceed the experts' $f$-ranges, whereas in 6 cases it leads to $f$-ranges that fall below the experts' $f$-ranges. The average overlap values obtained by the GA-VBAC and the experts reached $92.5 \%$ and $91.8 \%$ respectively. These results imply that the GA-VBAC framework is capable of learning from a single training image and of obtaining high delineation accuracies on independent sets of images. The obtained delineation accuracy is comparable to or even higher than the delineation accuracies obtained by individual expert radiologists.

The interobserver variability as quantified by the coefficient of variation [51] ranges between $1.0 \%$ and $9.8 \%$. The coefficient of variation of the overlap values obtained with GA-VBAC ranges between $1.0 \%$ and $3.3 \%$, and in all the cases, it was lower or at most equal to the coefficient of variation of the experts.

Figure 6 illustrates the delineations for two indicative thyroid nodule cases, as drawn by GA-VBAC (Fig.6b,d) in comparison to the corresponding ground truth 
delineations (Fig.6a,c). The average overlap value per generation estimated over the 44 cross validation repetitions of the GA-VBAC, for the two thyroid ultrasound images are illustrated in Fig. 7. In Case 17 the average overlap value achieved at convergence was $95.0 \%$, and in Case 32 it was $92.2 \%$. In both cases, the convergence was reached in less than 50 generations.

\section{[FIGURE 6]}

The average time required for the execution of the VBAC algorithm is approximately $93 \mathrm{~s}$. The total training time of the GA-VBAC framework is $38.7 \mathrm{~h}$, which is justified considering the population size $R$ and the maximum number of generations $G_{\max }$ used in the experiments. This time is reduced to $18.9 \mathrm{~h}$ by storing the fitness values so as they do not have to be recalculated for the chromosomes maintained in the population. It should be noted that: (a) if one had to follow the naive approach of exhaustive search in the parameter space, the execution time required would be almost 700 times more, and (b) the GA-VBAC framework has to be applied only once, for training. The resulting set of optimal parameters $\left(k^{+}, k^{-}, \alpha\right)$ may be applied for the delineation of thyroid nodules in other thyroid ultrasound images acquired from the same ultrasound imaging device with the same settings. This means that for each new image, only the execution time of VBAC is required.

\section{Conclusions}

We can draw a few important inferences for the proposed GA-VBAC framework. This novel framework embodies VBAC combined with a GA. VBAC is a level set segmentation approach that copes with the intensity inhomogeneity of thyroid ultrasound images by considering variable background image regions. The GA has been employed for efficient automatic tuning of VBAC parameters to an optimal set of values. The combination of VBAC with GAs leads to accurate delineation of nodules in thyroid ultrasound images. The accuracy it provides is comparable to or even higher than the delineation accuracies obtained by expert radiologists. 
The results show that the interobserver variability of the expert radiologists is higher than the variability of the overlap values obtained with GA-VBAC. Therefore, the proposed framework offers a tool for objective medical judgment of the thyroid nodule. Moreover, it provides the physicians with a second opinion, without requiring technical skills and time-consuming manual interaction for parameter tuning.

In our future work, the proposed GA-VBAC framework could be enhanced by speeding up the training phase, which will contribute to the feasibility of training with multiple ultrasound images. Moreover, it could be embedded within an integrated system that will combine heterogeneous information to support thyroid nodule diagnosis.

\section{[FIGURE 7]}

Acknowledgments. We would like to thank Dr. N. Dimitropoulos M.D. Radiologist, and EUROMEDICA S.A., Greece, for the provision of the thyroid ultrasound images and their contribution in the evaluation of the results. This work was supported by the Greek General Secretariat of Research and Technology and the European Social Fund, through the PENED 2003 program (grant no. 03-ED-662).

\section{References}

1. AACE Clinical Practice Guidelines for the Diagnosis and Management of Thyroid Nodules, Endocrine Practice, vol. 2(1), pp. 78-84, 1996

2. S.V.B. Jardim and M.A.T. Figueiredo, "Automatic contour estimation in fetal ultrasound images,” in Proc. IEEE Int. Conf. Im. Proc., vol. 2, pp.1065-1068, 2003.

3. New York Thyroid Center page: http://cpmcnet.columbia.edu/dept/thyroid/.

4. E. Papini, R. Guglielmi, A. Bianchini, A. Crescenzi, S. Taccogna, F. Nardi, C. Panunzi, R. Rinaldi, V. Toscano and C.M. Pacella, "Risk of malignancy in nonpalpable thyroid nodules: predictive value of ultrasound and color-doppler features," The Journal of Clinical Endocrinology and Metabolism, vol. 87(5), pp. 1941-1946, 2002.

5. Y. Zimmer, R. Tepper and S. Akselrod, "A two-dimensional extension of minimum cross entropy thresholding for the segmentation of ultrasound images," Ultrasound in Medicine and Biology, vol. 22, pp. 1183-1190, 1996. 
6. R. Adams and L. Bischof, "Seeded region growing," IEEE Trans. Patt. Anal. Mach. Intell., vol. 16(6), pp. 641- 647, 1994.

7. R. Pohle and K. Toennies, "Segmentation of medical images using adaptive region growing," in Proc. SPIE Med. Im., vol. 4322, pp. 1337-1346, 2001.

8. X. Hao, C. Bruce, C. Pislaru and J.F. Greenleaf, "A novel region growing method for segmenting ultrasound images," in Proc. IEEE Int. Ultrasonics Symposium, vol. 2, pp. 1717-1720, 2000.

9. C. Kotropoulos and I. Pittas, "Segmentations of ultrasonic images using support vector machines," Pattern Recognition Letters, vol. 24. Elsevier Science, pp. 715-727, 2003.

10.D. Boukerroui, O. Basset, N. Guerin and A. Baskurt, "Multiresolution texture based adaptive clustering algorithm for breast lesion segmentation," European Journal of Ultrasound, vol. 8, pp. 135-144, 1998.

11.L. Fan, G.A. Braden and D.M. Herrington, "Nonlinear wavelet filter for intracoronary ultrasound images," in Proc. of Annual Meeting on Computers in Cardiology, pp. 41-44, 1996.

12.J.G. Thomas, R.A. Peters and P. Jeanty, "Automatic segmentation of ultrasound images using morphological operators,” IEEE Trans. Med. Im., vol. 10, pp. 180-186, 1991.

13.T. Heckman, "Searching for contours," in Proc. SPIE, vol. 2666, pp. 223-232, 1996.

14.B. Solaiman, C. Roux, R.M. Rangayyan, F. Pipelier and A. Hillion, "Fuzzy edge evaluation in ultrasound endosonographic images," in Proc. Canadian Conf. Elec. Comp. Eng., pp. 335338, 1996.

15.C.M. Chen, H.H.S. Lu and A.T. Hsiao, "A dual-snake model of high penetrability for ultrasound image boundary extraction," Ultrasound in Medicine and Biology, vol. 27(12), pp. 1651-1665, 2001.

16.T. Haenselmann, C. Schremmer and W. Effelsberg, "Wavelet based semi-automatic segmentation of image objects," Signal and Im. Proc., pp. 387-392, 2001.

17.A. Blake and M. Isard, "Active contours: The application of techniques from graphics, vision, control theory and statistics to visual tracking of shapes in motion," Springer Verlag, Berlin Heidelberg New York, 1999.

18.N. Paragios and R. Deriche, "Geodesic active regions and level set methods for supervised texture segmentation,” Int. Jour. Comp. Vis., vol. 46(3), pp. 223-247, 2002.

19.T. McInerney and D. Terzopoulos, "Deformable models in medical images analysis: a survey,” Med. Im. Anal., vol. 1(2), pp. 91-108, 1996.

20.J.H. Lee and C.J. Lin, “Automatic model selection for support vector machines," Visual Inf. and Inf. Systems, pp. 37-48, 2000. 
21.S. Theodoridis and K. Koutroumbas, "Pattern recognition,” Academic Press, 1998.

22.J.S. Suri, K. Liu, S.N. Laxminarayan, X. Zeng and L. Reden, "Shape recovery algorithms using level sets in 2-D/3-D medical imagery: a state-of-the-art review," ?? ? Trans. Inf. Tech. in Biomed., vol. 6(1), pp. 8-28, 2002.

23.E. Angelini, J. Holmes and A. Laine, "Segmentation of RT3D ultrasound with implicit deformable models without gradients," in Proc. ISPA, vol. 2, pp. 711-716, 2003.

24.D. Honigmann, J. Ruisz and H. Pottmann, "Fast model based segmentation of ultrasound data using an active image," in Proc. IEEE Int. Symp. Biomed. Imaging, pp. 225-228, 2002.

25.N. Lin, W. Yu and J.S. Duncan, "Combinative multi-scale level set framework for echocardiographic image segmentation,” Med. Im. Anal., vol. 7, pp. 529-537, 2003.

26.T.F. Chan and L.A. Vese, “Active contours without edges," IEEE Trans. Im. Proc., vol. 7, pp. 266-277, 2001

27.M.A. Savelonas, D.E. Maroulis, D.K. Iakovidis, S.A. Karkanis and N. Dimitropoulos, “A variable background active contour model for thyroid nodule detection in ultrasound images,” in Proc. IEEE Int. Conf. Im. Proc., vol. 1, pp.17-20, 2005.

28.M. Kass, A. Witkin and D. Terzopoulos, "Snakes: Active contour models," Int. Journal of Comp. Vis., vol. 8(2), Springer, Netherlands, pp. 321-331, 1998.

29.A.K. Jain, Y. Zhong and S. Lakshmanan, "Object matching using deformable models," IEEE Trans. Patt. Anal. Mach. Intell., vol. 18(3), pp. 267-278, 1991.

30.O.V. Larsen, P. Radeva and E. Marti, "Guidelines for choosing optimal parameters of elasticity for snakes," in Proc. Comp. Anal. of Im. and Patt., pp. 106-113, 1995.

31.K.F. Lai and R.T. Chin, "Deformable contours: Modeling and extraction,” IEEE Trans. Patt. Anal. Mach. Intell., vol. 17(11), pp. 1084-1090, 1995.

32.D.H. Chen and Y.N. Sun, "A self-learning segmentation framework - the Taguchi approach," Computerized Medical Imaging and Graphics, vol. 24(5), pp. 283-296, 2000.

33.J.M. Morrel and S. Solimini, "Segmentation of images by variational methods: a constructive approach,” Revista Matematica Universidad Computense de Madrid, vol.1, pp. 169-182, 1988.

34.S. Osher and J. Sethian, "Fronts propagating with curvature- dependent speed: algorithms based on the Hamilton-Jacobi formulations," Journal of Computational Physics, vol. 79, pp. 12-49, 1988.

35.D.E. Goldberg, "Genetic algorithms in search, optimization and machine learning," Addison-Wesley: Reading, MA, 1989.

36.J.J. Grefenstette, "Optimization of control parameters for genetic algorithms," IEEE Trans. Syst. Man, and Cyber., vol. 16(1), pp. 122-128, 1986. 
37.L.M. Schmitt, "Theory of genetic algorithms II: Models for genetic operators over the string-tensor representation of populations and convergence to global optima for arbitrary fitness function under scaling,” Theoretical Computer Science, vol. 310, pp. 181-231, 2004.

38.S.H. Min, J. Lee and I. Han, "Hybrid genetic algorithms and support vector machines for bankruptcy prediction,” Expert Systems with Applications, vol. 31(3), Elsevier Science, pp. 652-660, 2006.

39.X.M. Zhao, Y.M. Cheung and D.S. Huang, "A novel approach to extracting features from motif content and protein composition for protein sequence classification," Neural Networks, vol. 18., Elsevier Science, pp. 1019-1028, 2005.

40.V.P Plagianakos, G.D. Magoulas and M.N. Vrahatis, "Tumor detection in colonoscopic images using hybrid methods for on-line neural network training," in Proc. of Int. Conf. Neural Networks and Expert Systems in Medicine and Healthcare, pp. 59-64, 2001.

41.G. Pignalberi, R. Cucchiara, L. Cinque and S. Levialdi, "Tuning range segmentation by genetic algorithm,” EURASIP Journal Appl. Sig. Proc., vol. 8, pp. 780-790, 2003.

42.M.R. Kaus, S.K. Warfield, F.A. Jolesz and R. Kikinis, "Segmentation of meningiomas and low grade gliomas in MRI," in Proc. Int. Conf. Medical Image Computing and ComputerAssisted Intervention, pp. 1-10, 1999.

43.G. Syswerda, "A study of reproduction in generational and steady state genetic algorithms," Foundations of Genetic Algorithms, G.J.E. Rawlings, San Mateo: Morgan Kaufmann, pp. 94-101, 1999.

44.D.E. Goldberg, B. Korb and K. Debb, "Messy genetic algorithms: motivation, analysis and first results," Complex Systems, vol. 3(5), pp. 493-530, 1989.

45.A.E. Eiben, "Multiparent Recombination in evolutionary computing, advances in evolutionary computing," Natural Computing Series, Springer Verlag, Berlin Heidelberg New York, pp. 175-192, 2002.

46.K.M. van Kemenade and A.E. Eiben, "Multi-parent recombination to overcome premature convergence in genetic algorithms," in Proc. Dutch Conference Art. Intell., pp. 137-146, 1995.

47.T. Bäck, "Optimal mutation rates in genetic search," in Proc. Int. Conf. Genetic Algorithms, pp. 2-8, 1993.

48.G. Rudolph, "Convergence analysis of canonical genetic algorithms,” IEEE Trans. Neural Networks, vol. 5, pp. 96-101, 1994.

49.D.E. Goldberg, "Sizing population for serial and parallel genetic algorithms," in Proc. of Int. Conf. Gen. Alg., pp. 70-79, 1989. 
50.T. Bäck, U. Hammel and H.P. Schwefel, "Evolutionary computation: Comments on the history and current state," IEEE Trans. Evol. Comp., vol. 1(1), pp. 3-17, 1997.

51.H.H. Woltjer, "The intra- and interobserver variability of impedance cardiography in patients at rest during exercise,” Physiol. Meas., vol. 17, pp. 171-178, 1996. 